\title{
Introduction to Pulmonary Aspergillosis
}

\author{
Dong-Na Su, Chen Qiu, Pu-Xuan Lu, and Shi-Ping Wu
}

Aspergillus is categorized into fungus that is extensively existing in the natural world. Aspergillosis is an infectious, allergic, and toxic disease caused by aspergillus. Pulmonary aspergillosis is caused by inhalation of airborne spores of aspergillus. Based on the immune state and potential pulmonary basic disease, the spores of aspergillus can cause different types of lung lesions, therefore different clinical manifestations and imaging demonstrations. Due to the extensive existence of the risk factors contributing to the occurrence of pulmonary aspergillosis, such as the prevalence of HIV/AIDS and tuberculosis, wide use of glucocorticoid, and wide application of organ transplantation as well as increasingly improved diagnostic techniques, the incidence and detection rate of pulmonary aspergillosis has been increasing recently. The disease has been fatal infectious disease in immunocompromised patients [1]. Due to the improved diagnostic technique, pulmonary aspergillosis is clinically diversifying, which presents challenges to the clinicians and specialists. Therefore, it is vitally important for clinicians and specialists to know about the key points for its diagnosis and the therapeutic principles.

Pulmonary aspergillosis is a bronchial and/or lung disease caused by aspergillus, mainly Aspergillus fumigatus. A joint effort was made by the European Society of Clinical Microbiology and Infectious Diseases (ESCMID) and European Respiratory Society (ERS) in the year 2015 to summarize previous research findings about chronic aspergillosis. The Chronic Pulmonary Aspergillosis: Rationale and Clinical guidelines for Diagnosis and Management (2015) [2] was hence published to replace the 2014 ECCMID Aspergillus Guideline [3]. Another effort was made by the
Infectious Diseases Society of America (IDSA) to summarize the relevant evidence of different types of aspergillosis based on the hot issues of its clinical diagnosis and management. And hence the new edition of the Guideline for Diagnosis and Management of Aspergillosis was published in 2016 [4] to replace the previous edition published in 2008 [5]. Starting with the basic theory, this chapter provides readers with a comprehensive understanding of the etiology, epidemiology, pathology and pathogenesis, clinical manifestations, diagnosis, and treatment of pulmonary aspergillosis in a short period of time. At the same time, it also lays a solid theoretical foundation for readers to correctly analyze and understand the wonderful case after the chapter.

\section{References}

1. Cunha C, Aversa F, Lacerda JF, et al. Genetic PTX3 deficiency and aspergillosis in stem-cell transplantation. $\mathrm{N}$ Engl $\mathrm{J}$ Med. 2014;370:421-32.

2. Denning DW, Cadranel J, Beigelman-Aubry C, et al. Chronic pulmonary aspergillosis: rationale and clinical guidelines for diagnosis and management. Eur Respir J. 2016;47:45-68.

3. Godet CL, Philippe B, Laurent F, Cadranel J. Chronic pulmonary aspergillosis: an update on diagnosis and treatment. Respiration. 2014;88(2):162-74.

4. Patterson TF, Thompson GR III, Denning DW, et al. Practice guidelines for the diagnosis and management of aspergillosis: 2016 update by the Infectious Diseases Society of America. Clin Infect Dis. 2016;63(4):e1-60.

5. Walsh TJ, Anaissie EJ, Denning DW, et al. Treatment of aspergillosis: clinical practice guidelines of the Infectious Diseases Society of America. Clin Infect Dis. 2008;46:327-60.

D.-N. Su · C. Qiu $(\bowtie) \cdot$ S.-P. Wu

Second Clinical Medical College of Jinan University,

Shenzhen, Guangdong, China

P.-X. Lu

Centre for Chronic Diseases Control of Shenzhen,

Shenzhen, Guangdong, China 